1 Radcliffe Department of Medicine, University of Oxford, Oxford, UK

2 NIHR Central Commissioning Facility, Twickenham, UK

3 Advance $\mathrm{HE}$, London, UK

4 Centre for Statistics in Medicine, Nuffield Department of Orthopaedics, Rheumatology and Musculoskeletal Sciences, University of Oxford, Oxford, UK

5 Oxford Academic Health Science Centre, Oxford, UK

6 Nuffield Department of Primary Care Health Sciences, University of Oxford, Oxford, UK

Correspondence to: P V Ovseiko pavel.ovseiko@rdm.ox.ac.uk Cite this as: BMJ 2020;371:m3975 http://dx.doi.org/10.1136/bmj.m3975 Published: 26 October 2020

\title{
Effect of Athena SWAN funding incentives on women's research leadership
}

\section{Analysis shows that funding incentives can work and more funders should trial them, say Pavel $\mathbf{V}$ Ovseiko and colleagues}

Pavel V Ovseiko, ${ }^{1}$ Mark Taylor, ${ }^{2}$ Ruth E Gilligan, ${ }^{3}$ Jacqueline Birks, ${ }^{4}$ Leena Elhussein, ${ }^{4}$ Mike Rogers, ${ }^{2}$ Sonja Tesanovic, ${ }^{2}$ Jazmin Hernandez, ${ }^{2}$ Glenn Wells, ${ }^{5}$ Trisha Greenhalgh, ${ }^{6}$ Alastair M Buchan ${ }^{1}$

"Is it difficult being a woman scientist?" the biochemist Dorothy Crowfoot Hodgkin was asked at high table dinner in an Oxford college by the man sitting next to her. "Not since I won the Nobel Prize," she replied. ${ }^{1}$ In 1964, the British press had reacted to her award with the headlines: "Oxford housewife wins Nobel" and "British woman wins Nobel Prize-£18 750 prize to mother of three." While such overtly sexist treatment of female scientists by the media is now rare, progress towards gender equality in universities has been astonishingly slow.

A UK parliamentary inquiry into women in scientific careers found that with only $17 \%$ of professors in science, technology, engineering, and mathematics (STEM) in 2011-12 women were still under-represented at senior levels across all STEM disciplines. ${ }^{3}$ Concerned with the sustainability of increasing the scientific workforce, the inquiry concluded that efforts to inspire more women into science were wasted if they were subsequently disadvantaged compared with men and recommended that universities should do more to support and retain women in scientific careers. ${ }^{3}$

To accelerate women's advancement and leadership, the UK's National Institute for Health Research (NIHR) introduced an innovative policy intervention in 2011 linking its research funding to the implementation by universities of gender equality action plans through the Athena SWAN (Scientific Women's Academic Network) charter (box 1). We examine the effect of this policy on women's research leadership in NIHR funded research and theorise how such incentives may work.

\section{Box 1: Athena SWAN charte}

The Athena SWAN charter provides a peer review framework for developing action plans and gaining recognition for the advancement of gender equality in higher education and research. The charter was established in 2005 under the ownership of the non-profit organisation Equality Challenge Unit (now Advance HE), evolving from the work of the Athena Project, set up to promote diversity in UK science, and the Scientific Women's Academic Network (SWAN).

Institutions that commit to the charter establish self-assessment teams to collect and analyse evidence, identify priorities, and develop action plans using a comprehensive framework for supporting and advancing women's careers 4 :

- Keycareer transition points-Recruitment, induction, promotion, research excellence
- Career development-Training, appraisal and development review, support for career progression, support for research grant applications

- Flexible working and managing career breaks-Cover and support for maternity and adoption leave; maternity return rate; uptake of paternity, shared parental, adoption, and parental leave; flexible working; transition from part-time back to full-time work after career breaks

- Organisation and culture-Culture; human resources policies; proportion of heads of school, faculty, or department by gender; representation of men and women on committees; participation on influential external committees; committee workload; policies, practices, and procedures; workload model; timing of meetings and social gatherings; visibility of role models; outreach activities.

The framework is applied to both institutions and departments. Institutions focus on the institutional structures and policies to enable change, whereas subject departments act as the agents of change by improving hiring, retention, and promotion decisions, workplace practices, and organisational culture at discipline level.

Institutions and departments apply to have their applications and action plans peer reviewed by subject specific panels of academics, experts, and Athena SWAN professionals from other participating institutions against the self-assessments presented and good practice in the field. Applicants receive constructive feedback and awards commensurate with the committed effort and outcomes ${ }^{4}$ :

- Bronze award requires an assessment of gender equality and the issues facing the institution or department plus a four year action plan to deal with these

- Silver award recognises the successful implementation of the action plan and its demonstrable impact

- Gold award recognises beacons of achievement in gender equality and champions in promoting good practice in the wider community

\section{National translational research infrastructure}

With the aim of creating a national infrastructure for translating scientific breakthroughs into new treatments for patients, NIHR has awarded £2.2bn (€2.5bn; \$2.9bn) of UK taxpayers' funding for translational research to biomedical research centres and units-partnerships between NHS organisations and universities. The strategic objectives, scope, and magnitude of funding of these centres have parallels 
with the Clinical and Translational Science Awards Program of the US National Institutes of Health. ${ }^{5}$

Biomedical research centres cover several disease, organ system, technology, and activity based research themes (eg, cancer, cardiovascular disease, genomics, and patient and public involvement) with multiple investigators in each theme. Centres are led by a director (senior leader) responsible for the strategy and coordination of research across themes and by theme leads (mid-level leaders), who have similar responsibilities across research topics within their theme.

Funding awards are based on peer review by an international selection panel of senior researchers and informed by bibliometrics. ${ }^{6}$ The panel considers applicants' research strategy, research facilities, budget, narrative track record of success in translational research, publications, grants, and the career history of the proposed director and theme leads, including a narrative description of why they are at the forefront of their field internationally and a list of publications over the past five years.

\section{Funding incentives for women in science}

During the 2011 competition for research centre funding, Sally Davies, then director general of research and development and chief scientific adviser at the Department of Health, sought to encourage universities to accelerate women's advancement and leadership in science. She stated that in the 2016 round of the competition the NIHR did not expect to shortlist any research centre where the academic partners had not achieved at least the Athena SWAN silver award.
NIHR's policy intervention provided sufficient incentives for universities to develop and implement gender equality action plans. This coincided with a 10-fold increase in the number of medicine related Athena SWAN silver or gold awards in 2016 (one gold and 69 silver awards) compared with 2011 (seven silver awards). Moreover, several major funders and science organisations in Ireland, Australia, the United States, and Canada subsequently adopted modified versions of the Athena SWAN framework contexts (see supplementary data on bmj.com for further details).

NIHR removed the requirement for applicants to hold Athena SWAN awards in 2020, partly to reduce administrative burden during the covid-19 pandemic. It stated: "we believe that use of the [Athena SWAN] charter has led to the greater embedding of gender equality practice" and that the future focus for NIHR would be on the broader commitment to all dimensions of equality, diversity and inclusion. ${ }^{7}$ Although Athena SWAN awards are no longer required, applicants can still choose to evidence the relevant elements of this new broader commitment through the awards.

\section{Effect of funding incentives}

After the introduction of the Athena SWAN incentives, the proportion of female theme leads increased to 24\% (43/177) in 2016 from 8\% in both 2006 (8/105) and 2011 (15/200). The proportion of women in senior director positions also increased from $11 \%(3 / 28)$ and 10\% (3/31) in 2006 and 2011 to 15\% (3/20) in 2016 (fig 1 and supplementary data on bmj.com). The incentives seem to have increased the number of female theme leads but not the number of female directors.

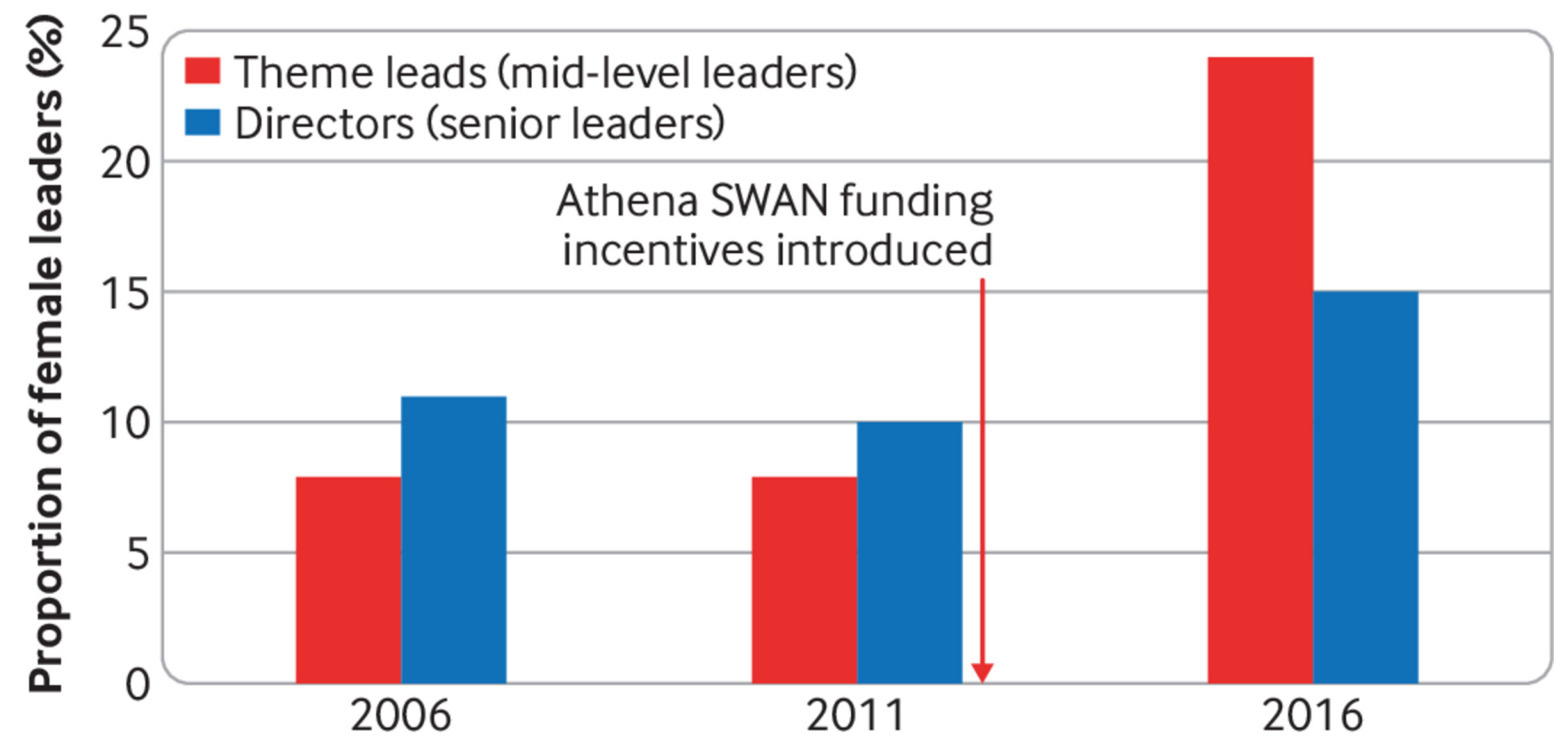

Fig 1 | Percentages of female research leaders

Before the introduction of the Athena SWAN funding incentives the proportion of funding obtained by female theme leads was $5 \%$ in 2006 and $4 \%$ in 2011. This increased to $21 \%$ in 2016. The proportion of funding obtained by female directors increased from $2 \%$ in both 2006 and 2011 to 4\% in 2016 (fig 2) but remained much lower than for male directors (see supplementary data for more detail). 


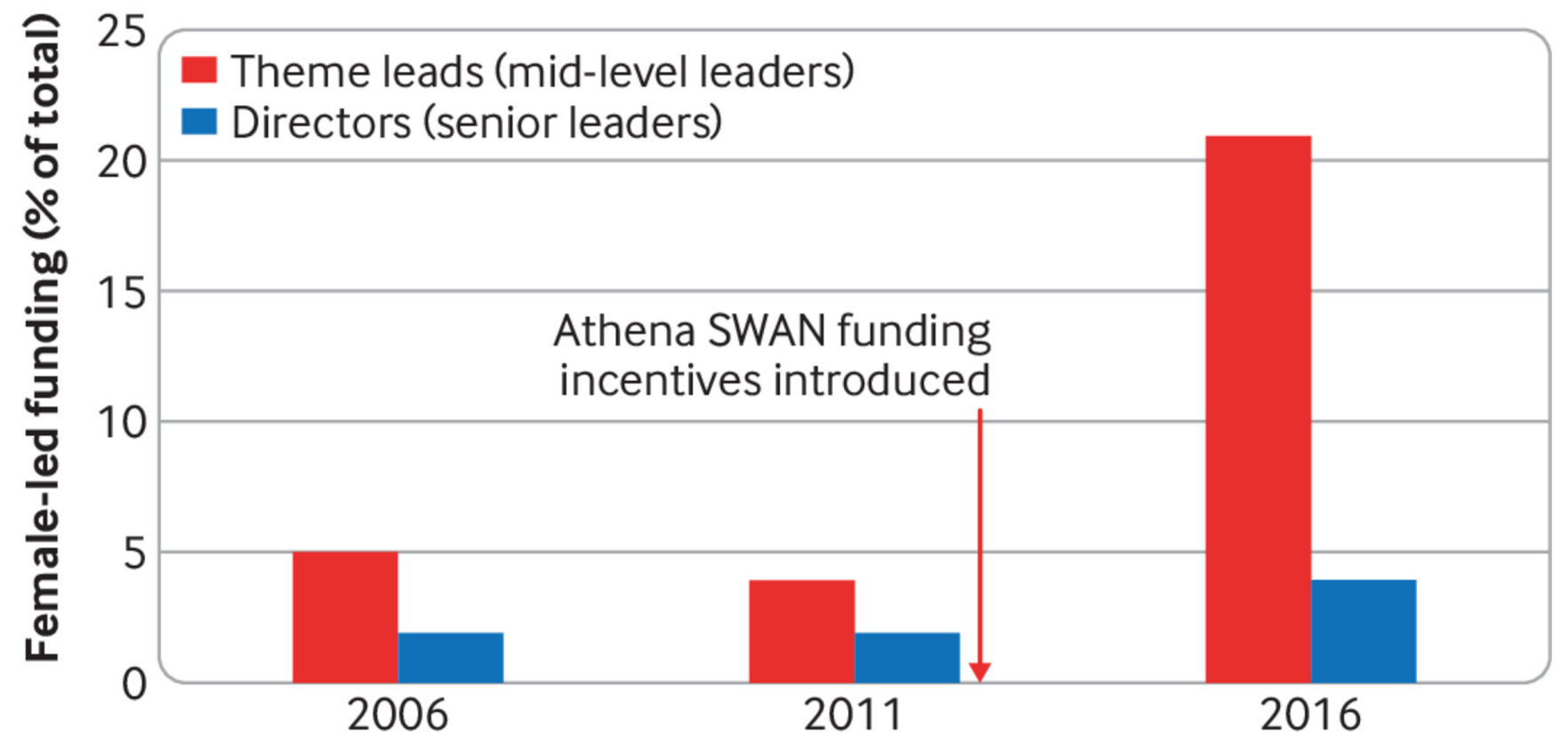

Fig 2 | Percentages of funding obtained by female research leaders

\section{Leaky pipelines}

One possible confounder for gender disparity in leadership positions is the pipeline effect. It is often suggested that once there are sufficient numbers of women entering universities and they are not discriminated against for admission into the pipeline, the under-representation of women at all levels in academic medicine will gradually disappear. To test this theory we estimated admission into the academic medicine pipeline using scientific age-that is, the number of years after the first publication. The scientific age of female and male theme leads ( 27 and 28 , respectively) and directors (27 and 30, respectively) was similar in 2016 (see supplementary data for more detail). Assuming the leads' first publication occurred in the first year after graduation, they entered medical school around 1980 when female admissions to UK medical schools were already $40 \% .{ }^{8}$ Given that the proportions of female theme leads and directors are much lower than $40 \%$, the pipeline appears to be leaking.

When we examined a leadership pipeline within research centres, we found that in $201665 \%$ (13/20) of directors and 40\% (71/177) of theme leads had held these roles in previous rounds and that more men than women had repeated leadership terms (see supplementary data for more detail). A disproportionately large pool of incumbent male leaders who seek repeated leadership terms more often than female leaders may partially explain the dearth of female directors.

We also examined gender balance on the international selection panel for research centre awards. In 2006 and 2011, the panels were all male but in 2016, 20\% (2/10) of panel members were women. However, we could not investigate the possible effects of this change empirically or draw conclusive inferences from previous studies.

Whereas some studies reported gender bias in peer review, ${ }^{9}$ others found no association between the applicant's gender and the reviewers' gender. ${ }^{10}$ One natural experiment suggested that gender equity in funding could be increased by focusing peer review on science rather than scientists. ${ }^{11}$
Although there could still be other unmeasured confounders such as structural changes in the number of centres and their research themes, our analysis suggests that the introduction of the Athena SWAN incentives contributed to the accelerated advancement of women to theme lead positions and gender equity gains in funding. Data from the 2021 funding round will help establish whether there is a causal link.

\section{Critical actors}

Our analysis shows the salience of "critical actor" leaders over "critical mass" for initiating change towards more gender equitable research leadership. Critical mass theory predicted that once the proportion of female academics reached $30-35 \%$, changes in the sociocultural environment would trigger an increase in women's leadership. However, recent research suggests that the promise of critical mass should be abandoned in favour of critical actors-"women and men, who individually and collectively have the commitment and power to create gender equitable cultures." 12

Sally Davies and NIHR were collective critical actors in introducing the Athena SWAN funding incentives, which were associated with important changes in NIHR funded research. Likewise, many committed leaders and the major national funders and science organisations acted collectively to lead the adoption of Athena SWAN principles globally.

Athena SWAN requires time to affect the numbers of female scientists because it seeks structural and cultural changes for all faculty and staff. ${ }^{13}$ Although Athena SWAN has been shown to be effective in challenging discrimination and bias, improving women's visibility and leadership skills, and initiating structural and cultural changes, ${ }^{14-17}$ it has limitations in tackling longstanding tenure, power, and pay imbalances in the short term. ${ }^{18} 19$ Unintended consequences have also been reported with the project, such as perceptions of administrative burden, ${ }^{20}$ women undertaking a disproportionate amount of work, ${ }^{21}$ and gender taking priority over race and class. ${ }^{22}$ 
Effective implementation of Athena SWAN seems to require sufficient commitment, time, resources, and professional expertise. The rise in women's research leadership in NIHR funded centres contrasts with previous research in 35 medical schools that showed no changes in gender balance among faculty two years after the introduction of the funding incentives. ${ }^{18}$ NIHR funded centres had more favourable conditions and sufficient time to implement four year action plans. The linkage of Athena SWAN to the $£ 816 \mathrm{~m}$ funding scheme incentivised universities to commit time of leaders, faculty, and staff, invest internal resources, and employ equality and diversity professionals to implement action plans in friendly competition with their peers. ${ }^{13} 15$

\section{Realising the benefits of gender equity}

The case for advancing gender equity in research is compelling. Equitable participation of all genders in research is imperative to social progress and legitimisation of public support for science. ${ }^{23}$ Drawing on the talents of all genders is also necessary for increasing the sustainability of the scientific workforce. ${ }^{3}$ Evidence suggests that gender diversity in groups is associated with greater problem solving ${ }^{24}$ and higher quality research. ${ }^{25}$ A gender equitable scientific workforce can therefore enhance the quality of science. As women pursue collaboration and interdisciplinary research more often than men, ${ }^{26} 27$ gender equity can also improve team science. Moreover, health research led by women more often investigates sex and gender related variables than does research led by men. ${ }^{2829}$ Gender equity in research leadership can therefore increase the scientific rigour and relevance of health research to women's health.

Funders can advance gender equity in research through policy interventions and funding incentives. For example, the US National Institutes of Health championed inclusion of women and minority groups in clinical trials as research beneficiaries. ${ }^{30}$ The Canadian Institutes of Health Research led the world in integrating sex and gender in research as variables to reduce sex and gender bias among research beneficiaries. ${ }^{30}$ From 2021, the European Commission will require all public bodies applying to Horizon Europe-the European flagship $€ 81$ bn research and innovation funding programme-to have gender equality action plans. ${ }^{31}$ More funders should assume the role of critical actor in trialling policy interventions and funding incentives for gender equity.

\section{Evaluating interventions and incentives}

Given the compelling case for gender equity and a lack of conclusive evidence on what works, for whom, and in what circumstances, we recommend evaluating the effect of Athena SWAN longitudinally using quasi-experimental designs, investigating any rare or unintended consequences, and testing its efficacy for other dimensions of diversity and inclusion. NIHR should consider the pros and cons of term limits for research leaders: refreshing leadership, encouraging innovation, and increasing diversity versus introducing disruption and inhibiting the development of expertise. ${ }^{32}$ Finally, NIHR can experiment with the gender composition of interview panels for research centre funding and facilitate evaluation by including in non-blind applications leaders' gender and previous leadership terms.

\section{Key messages}

- Gender equity in research can contribute to social progress, scientific workforce sustainability, and the quality of science

- Research funders can be critical actors in advancing gender equity through policy interventions and funding incentives
- Linking NIHR funding to Athena SWAN gender equality action plans has been associated with a rise in the number of women in mid-level leadership positions and the proportion of funding going to women

- More research funders should trial policy interventions and funding incentives for women in science and evaluate their effect

Contributors and sources: PVO and MT conceived of the article. MT, MR, ST, JB, and REG participated in data collection. JB and LE provided statistical analysis, PVO, MT, MR, REG, JB, GW, TG, and AMB all participated in the drafting and reviewing of the article. PVO wrote the first draft of the manuscript, provided the figures, and is the guarantor.

Competing interests: We have read and understood BMJ policy on declaration of interests and declare the following interests: PVO, JB, LE, and TG are supported by the National Institute for Health Research (NIHR) Oxford Biomedical Research Centre, grant BRC-1215-20008 to the Oxford University Hospitals NHS Foundation Trust and the University of Oxford; PVO is a member of the NIHR advisory group on equality, diversity, and inclusion; a member of the Advance HE Athena SWAN governance committee; and a member of the Athena SWAN self-assessment team, Radcliffe Department of Medicine, University of Oxford. AMB was supported by the NIHR senior investigator award; MT serves on BMJ patient panel. MT, MR, ST, and JH are employed by NIHR; REG is employed by Advance HE and works on Athena SWAN charter. The views expressed are those of the authors and not necessarily those of the NHS, the NIHR, or the Department of Health.

Provenance and peer review: Not commissioned; externally peer reviewed.

We thank Wafa El-Adhami, Karine Morin, Shirley M Malcom, Beth Ruedi, Syed Ghulam Sarwar Shah.

Spokes Symonds A. Also-rans: the injustice of history. Robert Boyd Publications, 2014.

Dorothy Hodgkin: The only British woman to win a Nobel science prize gets a doodle. Guardian 2014 May 12. https://www.theguardian.com/technology/2014/may/12/google-doodle-honoursbiochemist-dorothy-hodgkin.

House of Commons Science and Technology Committee. Women in scientific careers. Sixth report of session 2013-14. 2014. https://publications.parliament.uk/pa/cm201314/cmselect/cmsctech/701/701.pdf.

Equality Challenge Unit. Athena SWAN charter awards handbook. 2015. https://www.ecu.ac.uk/wpcontent/uploads/2015/05/ECU-Handbook-26.05.15-FINAL.pdf.

Soderquest K, Lord GM. Strategies for translational research in the United Kingdom. Sci Transl Med 2010;2:53cm28. doi: 10.1126/scitransImed.3001129 pmid: 20944087

6 Gunashekar S, Wooding S, Guthrie S. How do NIHR peer review panels use bibliometric information to support their decisions? Scientometrics 2017;112:1813-35.

doi: 10.1007/s11192-017-2417-8 pmid: 28804180

National Institute for Health Research. NIHR responds to the Government's call for further reduction in bureaucracy with new measures. 2020. https://www.nihr.ac.uk/news/nihr-responds-to-thegovernments-call-for-further-reduction-in-bureaucracy-with-new-measures/25633.

8 Edmunds LD, Ovseiko PV, Shepperd S, etal. Why do women choose or reject careers in academic medicine? A narrative review of empirical evidence. Lancet 2016;388:2948-58. doi: 10.1016/S0140-6736(15)01091-0 pmid: 27105721

9 Wennerås C, Wold A. Nepotism and sexism in peer-review. Nature 1997;387:341-3. doi: 10.1038/387341a0 pmid: 9163412

10 Mutz R, Bornmann L, Daniel HD. Does gender matter in grant peer review?: an empirical investigation using the example of the Austrian Science Fund. Z Psychol 2012;220:121-9. doi: 10.1027/2151-2604/a000103 pmid: 23480982

11 Witteman HO, Hendricks M, Straus S, Tannenbaum C. Are gender gaps due to evaluations of the applicant or the science? A natural experiment at a national funding agency. Lancet 2019;393:531-40. doi: 10.1016/S0140-6736(18)32611-4 pmid: 30739688

12 Helitzer DL, Newbill SL, Cardinali G, Morahan PS, Chang S, Magrane D. Changing the culture of academic medicine: critical mass or critical actors?] Women's Health (LarChmt) 2017;26:540-8. doi: 10.1089/jwh.2016.6019 pmid: 28092473

13 Kalpazidou Schmidt E, Ovseiko PV, Henderson LR, Kiparoglou V. Understanding the Athena SWAN award scheme for gender equality as a complex social intervention in a complex system analysis of Silver award action plans in a comparative European perspective. Health Res Policy Syst 2020;18:19. doi: 10.1186/s12961-020-0527-x pmid: 32059678

14 Ovseiko PV, Chapple A, Edmunds LD, Ziebland S. Advancing gender equality through the Athena SWAN charter for women in science: an exploratory study of women's and men's perceptions. Health Res Policy Syst 2017;15:12. doi: 10.1186/s12961-017-0177-9 pmid: 28222735

15 Ovseiko PV, Pololi LH, Edmunds LD, etal. Creating a more supportive and inclusive university culture: a mixed-methods interdisciplinary comparative analysis of medical and social sciences at the University of Oxford. Interdiscip Sci Rev 2019;44:166-91doi: 10.1080/03080188.2019.1603880

16 Graves A, Rowell A, Hunsicker E. An Impact Evaluation of the Athena SWAN Charter. 2019. https://www.ecu.ac.uk/wp-content/uploads/2019/08/Athena-SWAN-Impact-Evaluation-2019.pdf.

17 Xiao Y, Pinkney E, Au TKF, Yip PSF. Athena SWAN and gender diversity: a UK-based retrospective cohort study. BMJ Open 2020;10:e032915. doi: 10.1136/bmjopen-2019-032915 pmid: 32051310

18 Gregory-Smith I. Positive action towards gender equality: evidence from the Athena SWAN charter in UK medical schools. Br J Ind Relat 2018;56:463-83doi: 10.1111/bjir.12252 
19 Rosser SV, Barnard S, Carnes M, Munir F. Athena SWAN and ADVANCE: effectiveness and lessons learned. Lancet2019;393:604-8. doi: 10.1016/S0140-6736(18)33213-6 pmid: 30739697

20 Tzanakou C. Unintended consequences of gender-equality plans. Nature 2019;570:277. doi: 10.1038/d41586-019-01904-1 pmid: 31213693

21 Caffrey L, Wyatt D, Fudge N, Mattingley H, Williamson C, McKevitt C. Gender equity programmes in academic medicine: a realist evaluation approach to Athena SWAN processes. BMJ Open 2016;6:e012090. doi: 10.1136/bmjopen-2016-012090 pmid: 27609850

22 Bhopal K, Henderson H. Competing inequalities: gender versus race in higher education institutions in the UK. Educ Rev 2019:1-17.doi: 10.1080/00131911.2019.1642305

23 Fine C, Sojo V. Women's value: beyond the business case for diversity and inclusion. Lancet 2019;393:515-6. doi: 10.1016/S0140-6736(19)30165-5 pmid: 30739677

24 Hong L, Page SE. Groups of diverse problem solvers can outperform groups of high-ability problem solvers. Proc Natl Acad Sci U S A 2004;101:16385-9. doi: 10.1073/pnas.0403723101 pmid: 15534225

25 Campbell LG, Mehtani S, Dozier ME, Rinehart J. Gender-heterogeneous working groups produce higher quality science. PLoS One 2013;8:e79147. doi: 10.1371/journal.pone.0079147 pmid: 24205372

26 Smith-Doerr L. Women's work: gender equality vs. hierarchy in the life sciences. Lynne Rienner, 2004.

27 van Rijnsoever FJ, Hessels LK. Factors associated with disciplinary and interdisciplinary research collaboration. Res Policy 2011;40:463-72doi: 10.1016/j.respol.2010.11.001

28 Nielsen MW, Andersen JP, Schiebinger L, Schneider JW. One and a half million medical papers reveal a link between author gender and attention to gender and sex analysis. Nat Hum Behav 2017;1:791-6. doi: 10.1038/s41562-017-0235-x pmid: 31024130

29 Sugimoto CR, Ahn Y-Y, Smith E, Macaluso B, Larivière V. Factors affecting sex-related reporting in medical research: a cross-disciplinary bibliometric analysis. Lancet 2019;393:550-9. doi: 10.1016/S0140-6736(18)32995-7 pmid: 30739690

30 Heidari S, Babor TF, De Castro P, Tort S, Curno M. Sex and gender equity in research: rationale for the SAGER guidelines and recommended use. Res Integr Peer Rev 2016;1:2. doi: 10.1186/s41073-016-0007-6 pmid: 29451543

31 Kalpazidou Schmidt E, Ovseiko PV. Link Horizon Europe funding to real steps to gender equality. Nature 2020;584:525. doi: 10.1038/d41586-020-02430-1 pmid: 32814915

32 Austin JP. Is academic medicine ready for term limits?Acad Med 2020;95:180-3. doi: 10.1097/ACM.0000000000003020 pmid: 31577584 